\section{What are association formation models good for?}

\author{
JAN DE Houwer \\ Ghent University, Ghent, Belgium
}

Dickinson (2009) agrees with two of the core claims that were made in my target article (De Houwer, 2009): Associative learning effects (often) depend on (1) the formation of propositions and (2) the operation of nonautomatic processes. Whereas I derived these claims from propositional models of learning, Dickinson (2009) argues that they are also compatible with association formation models (AFMs). He also defends the position that only AFMs can explain "nonrational responses to associative experiences." In this comment, I question whether there is much value in continuing to cling to AFMs.

In the first part of his comment, Dickinson (2009) discusses earlier work (Dickinson, 1980) in which he put forward the hypothesis that many aspects of associative learning are driven by propositional knowledge. I fully subscribe to this hypothesis and acknowledge that Dickinson was one of the first to propose it in such an explicit manner. I do dispute, however, the claim that association formation models (AFMs) can account for the acquisition of propositional knowledge. A proposition can be seen as a qualified association between representations of events. It specifies not only that the events are related, but also how they are related. Whereas all AFMs incorporate assumptions about how organisms learn that events are related, I do not know any AFM that specifies how organisms acquire information about the way in which events are related. For instance, if there is a contingency between the occurrence of a disease and the presence of a substance in the blood, how would an AFM decide that the disease is an effect of the substance in the blood, or that the disease is the cause of the substance in the blood (see Waldmann \& Holyoak, 1992)? Until it is clarified how AFMs learn about the way in which events are related, one should not take seriously the claim that they can account for the acquisition of propositions. Propositional models, on the other hand, postulate that organisms form and evaluate propositions on the basis of other propositions that they entertain. It is precisely this core assumption that has led to the identification of many new determinants of associative learning, such as causal knowledge, instructions, and deductive reasoning (see De Houwer, 2009, for a review). Little attention was given to these determinants until the arrival of propositional models, because AFMs have little to say about them.
In the second part of his comment, Dickinson (2009) correctly points out that certain important AFMs incorporate the assumption that association formation is nonautomatic, in the sense of being dependent on attention and cognitive resources. Proponents of propositional models go a step further still by postulating that the process underlying associative learning (i.e., the formation and evaluation of propositions) is also nonautomatic with regard to the need for awareness and goals (see De Houwer, 2009). No current AFM shares the latter assumption. It is true, however, that all evidence for the nonautomatic nature of associative learning can easily be accommodated by AFMs simply by adding assumptions about the way in which the association formation process is nonautomatic. Although adding these assumptions would make the models more compatible with the existing evidence, it would also decrease their appeal. One of the reasons many researchers believe in the existence of association formation processes is precisely that these processes might account for seemingly automatic, irrational types of learning. If association formation processes are as nonautomatic as propositional reasoning, what is the use of assuming their existence? Also, there is no a priori reason to assume that association formation is a nonautomatic process. There are, however, good reasons to assume that propositional reasoning is a largely nonautomatic process. From this perspective, one could even argue that AFMs that emphasize the role of attention and cognitive resources are actually mathematical formalizations of propositional reasoning. They can be seen as a mathematical description of the functional operating principles of propositional reasoning, rather than as models of association formation (see also Mitchell, De Houwer, \& Lovibond, in press). In sum, there is little to be gained by assuming that association formation is a nonautomatic process.

The arguments of Dickinson (2009) that I have discussed so far were aimed at convincing us that association formation is not so different from propositional reasoning after all: Both can generate propositional knowledge, and both are nonautomatic in certain respects. I have argued against this conclusion and pointed out that it reduces the need to postulate the existence of association formation processes in the first place. Dickinson (2009) also presents a third argument that does emphasize the uniqueness of AFMs - namely, the fact that they are able to account for "nonrational responses to associative experiences" (p. 22). I have several problems with this argument. First, it seems to contradict his claim that association formation is nonautomatic. One could argue that there are several kinds of association formation processes, some automatic, some not. However, continuing to add different systems of learning is not parsimonious and renders the association

J. De Houwer, jan.dehouwer@ugent.be 
formation approach even less falsifiable than it already is. Second, as I have argued in my target article, there is no need to assume the existence of an automatic association formation system. Propositional models can account for the available evidence on nonrational responses to associative experiences (see also Mitchell et al., in press). Dickinson (2009) correctly points out that I did not specify a mechanism by which propositional beliefs can lead to the automatic, seemingly irrational responses (e.g., sweating). Nevertheless, because simply instructing people about future events can also lead to such automatic, irrational responses, it is clear that such a mechanism must exist. How mind and body interact has been a heavily debated issue for hundreds of years already, one that I do not aspire to have the final say on. It remains to be seen whether the answer lies in the existence of an association formation mechanism, as Dickinson (2009) seems to argue, or in the impact of automatic processes involved in perception and memory (see De Houwer, 2009, note 8), or in still other processes. Those who decide to study whether AFMs do have unique merits should stop assuming by default that associative learning effects are driven by association formation and put forward valid and verifiable criteria for determining whether learning effects are due to association formation rather than to propositional reasoning.

\section{AUTHOR NOTE}

Preparation of this article was supported by Grant BOF/GOA2006/001 from Ghent University. I thank Tom Beckers for comments on a previous version of this article. Correspondence concerning this article should be addressed to J. De Houwer, Department of Psychology, Ghent University, Henri Dunantlaan 2, B-9000 Ghent, Belgium (e-mail: jan .dehouwer@ugent.be).

\section{REFERENCES}

De Houwer, J. (2009). The propositional approach to associative learning as an alternative for association formation models. Learning \& Behavior, 37, 1-20.

Dickinson, A. (1980). Contemporary animal learning theory. Cambridge: Cambridge University Press.

Dickinson, A. (2009). What are association formation models? Learning \& Behavior, 37, 21-24.

Mitchell, C. J., De Houwer, J., \& Lovibond, P. F. (in press). The propositional nature of human associative learning. Behavioral \& Brain Sciences.

Waldmann, M. R., \& HolyoaK, K. J. (1992). Predictive and diagnostic learning within causal models: Asymmetries in cue competition. Journal of Experimental Psychology: General, 121, 222-236.

(Manuscript received September 9, 2008; accepted for publication October 10, 2008.) 